\title{
Examining the use of antiviral prophylaxis for influenza outbreaks in residential aged care facilities in NSW, Australia
}

\author{
Wedyan Meshrekya,d, Daneeta Hennessyb,c, Robin Gilmourc, Sean \\ Tobin and Vicky Sheppeard ${ }^{c}$ \\ a NSW Public Health Training Program, NSW Health, Sydney, Australia \\ b NSW Biostatistics Training Program, NSW Health, Sydney, Australia \\ c Communicable Diseases Branch, Health Protection NSW, NSW Health, Sydney, Australia \\ d Corresponding author: wedyan.meshreky@gmail.com
}

\section{Article history}

Publication date: March 2020

Citation: Meshreky W, Hennessy D,

Gilmour R, Tobin S, Sheppeard V. Examining the use of antiviral prophylaxis for influenza outbreaks in residential aged care facilities in NSW, Australia. Public Health Res Pract. 2020;30(1):e29121904. First published: 10 April 2019. https://doi.org/10.17061/ phrp29121904

\section{Key points}

- Routine recommendations that New South Wales Public Health Units provide to residential aged care facilities (RACFs), about the use of prophylactic neuraminidase inhibitors (NAIs) during influenza outbreaks, vary across the state

- Delays in starting NAls and a low proportion of residents being administered prophylaxis are common in RACFs

- Interventions to support RACFs to appropriately administer NAls, identify outbreaks promptly, use line listings and increase uptake of staff vaccination rates may support future management of RACF influenza outbreaks

\section{Abstract}

Background: Influenza attack rates in closed population settings, such as residential aged care facilities (RACFs), can be more than $50 \%$ during annual epidemics. Uncertainty about the effectiveness of neuraminidase inhibitors (NAls) as prophylaxis for influenza outbreaks has led to variations in their use in RACFs in New South Wales (NSW), Australia.

Objectives: To examine the use of prophylactic NAIs by NSW RACFs for residents during influenza outbreaks in the 2015 influenza season.

Methods: A prospective cohort study of influenza outbreaks reported to NSW Public Health Units from 1 June 2015 - 31 October 2015.

Results: Eighty-eight RACFs reported influenza outbreaks; 86 were included in the study. Fifty-two RACFs used prophylactic NAls; 34 did not. The median time to start NAI prophylaxis from the onset date of the first case was 8.5 days (range 2-23). The average proportion of residents within a facility that received prophylaxis was 51\% (range 0.7-95).

Conclusion: Variations in the use of prophylactic NAls exist across RACFs. Earlier initiation of NAI prophylaxis, improved resident coverage where appropriate and other practice changes are recommended for the management of influenza outbreaks in RACFs. 


\section{Introduction}

Influenza is an acute, infectious respiratory viral infection that can be transmitted through respiratory droplets by sneezing, coughing or touching contaminated surfaces. ${ }^{1}$ Attack rates during annual epidemics can range from $5-20 \%$ in the general community to more than $50 \%$ in closed population settings such as residential aged care facilities (RACFs). In New South Wales (NSW), Australia, influenza infections increase and typically peak during the winter months (June-August). ${ }^{2}$

People aged 65 years and older are at increased risk of severe disease from seasonal influenza and are recommended to have an annual influenza vaccination. ${ }^{3}$ However, influenza vaccine efficacy is estimated to be at least $20 \%$ lower in this age group. ${ }^{4}$ As a result, this vulnerable population, especially people living in RACFs, are at increased risk of morbidity and mortality from influenza outbreaks, even when influenza vaccination rates are high.

In NSW, laboratory-confirmed influenza infection is a notifiable condition under the Public Health Act 2010. ${ }^{5}$ RACFs are not required to report influenza outbreaks under NSW public health legislation, but are encouraged to report suspected and confirmed influenza outbreaks to their local public health unit (PHU) to ensure optimum outbreak management and control. ${ }^{2}$

Neuraminidase inhibitors (NAls) are widely recommended in the treatment and prophylaxis of hospitalised patients with influenza, and other patients with influenza who are at risk of severe illness. ${ }^{6,7} \mathrm{~A}$ recent Cochrane Collaboration review of randomised controlled trials of NAls in healthy individuals with seasonal influenza recommended a review of the guidance on using NAls. This recommendation was based on the findings of small benefit of treatment compared with risk of harm. ${ }^{8}$ However, the same review concluded that prophylactic use of NAls reduced the risk of developing symptomatic influenza. In a separate meta-analysis of randomised controlled trials and observational studies, prophylaxis with oseltamivir was associated with significantly reduced odds of laboratory-confirmed influenza (odds ratio [OR] 0.11; 95\% confidence interval [Cl] 0.06, 0.20) compared with placebo or no therapy. ${ }^{9}$ Other observational studies have found a strong association between early use of influenza NAls for treatment and prophylaxis and effective outbreak control in RACFs. ${ }^{10-12}$

Consideration of the use of NAIs (specifically oseltamivir) is part of national guidelines for managing influenza outbreaks in RACFs. ${ }^{2}$ This consideration is an adjunct to measures such as infection control, restrictions on movement, and resident and staff vaccination, and is similarly recommended in comparable public health guidance documents of other countries. ${ }^{13-15}$ A clinician, often a resident's general practitioner, is required to prescribe NAls for residents. In some circumstances, PHU nursing staff may also prescribe NAls under medical supervision. $^{2}$
There have been no studies examining the use of NAls across all reported influenza outbreaks in NSW RACFs. In addition, uncertainty about the effectiveness of prophylactic NAls in RACF settings has led to variations in the routine recommendations that local PHUs make to RACFs about NAl use. ${ }^{16}$

This study examines the use of prophylactic NAls (specifically oseltamivir) by NSW RACFs for residents during influenza outbreaks in the 2015 influenza season (1 June 2015 - 31 October 2015).

\section{Methods}

We used a prospective cohort study design using data collected in the NSW Notifiable Conditions Information Management System (NCIMS). The NCIMS is a confidential database application used to manage the surveillance and routine reporting of diseases and conditions notifiable under the NSW Public Health Act 2010. As such, ethical approval for the study was not required.

Before the study period, an influenza outbreak investigation line listing template, which includes information about the case, room location, symptoms, vaccination, testing information and antiviral use, was updated to standardise collection of information about use of NAls during outbreaks. When an outbreak is reported to a PHU by an RACF, the PHU asks RACF staff to complete the line listing and email or fax the template to the relevant PHU. The PHU then enters the data into the NCIMS. All influenza outbreaks in NSW RACFs reported in the NCIMS between 1 June 2015 - 31 October 2015 were included in the study. Outbreaks were reviewed to assess completeness of data and identify any discrepancies in data entries. Influenza strain type was determined through routine laboratory reporting.

Only resident data were included in the analysis. Staff outcomes were excluded because information was often missing. The following definitions apply:

- Resident case: a person with an influenza-like illness

(ILI) with or without laboratory-confirmation of influenza

- Influenza outbreak: three or more epidemiologically linked cases of ILI in residents or staff of the facility within a 72-hour period, plus at least one case having a positive laboratory test for influenza (laboratoryconfirmed case) $)^{2}$

- Total number of residents at risk: number of residents registered in the RACF at the time of the outbreak regardless of area or room location.

All residents who were hospitalised or died during the outbreak, regardless of reported ILI symptoms, were included in the descriptive analysis. This was because of challenges in obtaining the cause of hospitalisation or death from RACFs. Further, elderly people may not present with fever or other typical influenza symptoms, so it is possible that residents experienced complications from influenza despite not displaying these symptoms. 
The final RACF cohort was divided into two groups: RACFs that started NAI prophylaxis, and RACFs that did not start NAI prophylaxis.

Data were analysed using SAS Enterprise Guide (Cary, NC: SAS Institute Inc; version 6.1).

\section{Results}

Eighty-eight RACF outbreaks were reported in the NCIMS between 1 June 2015 - 31 October 2015. Two RACFs had no information about whether or not NAls were used by the facility, so were excluded (Figure 1). Eighty-six
RACF outbreaks were included, affecting 9067 residents. Among residents, 1325 (14.6\%) were reported to have ILI symptoms, 145 (1.6\%) were hospitalised and $54(0.6 \%)$ died (Table 1). The most commonly reported ILI symptoms were cough, fever and lethargy. The average age of residents with laboratory-confirmed influenza was 85 years; 69\% were female. There were 533 (5.9\%) laboratory-confirmed influenza results reported among residents. Influenza A was the most common influenza type associated with RACF outbreaks during the study period (Table 2). Two RACFs reported their first case to be a staff member.

Figure 1. Reported use of neuraminidase inhibitors (NAIs) for influenza outbreaks in NSW residential aged care facilities (RACFs), 1 June 2015 - 31 October 2015

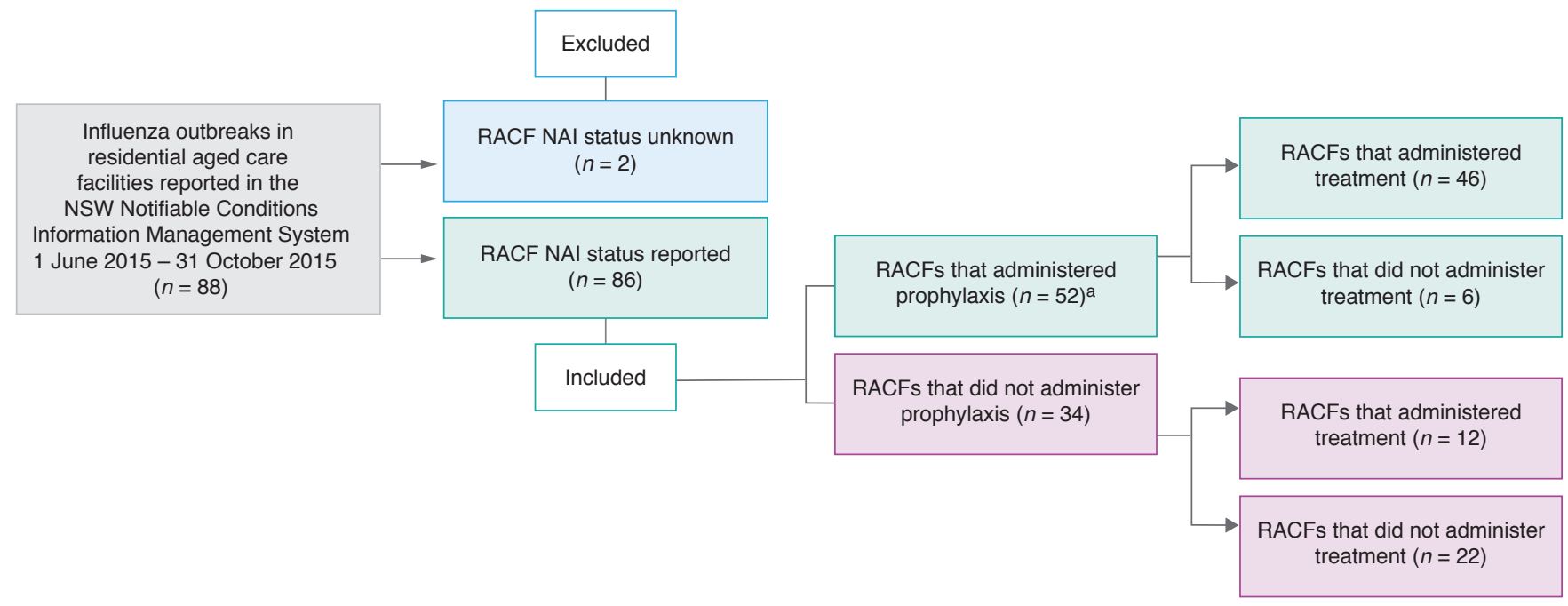

a Proportion of prophylaxis administered varied between RACFs

Table 1. Influenza outbreak characteristics in NSW residential aged care facilities, by neuraminidase prophylaxis, 1 June 2015 - 31 October 2015

\begin{tabular}{|c|c|c|c|c|c|c|c|}
\hline \multirow[b]{2}{*}{ Outbreak characteristics } & \multicolumn{3}{|c|}{$\begin{array}{l}\text { No prophylaxis } \\
\quad(n=34)\end{array}$} & \multicolumn{3}{|c|}{$\begin{array}{c}\text { Prophylaxis } \\
(n=52)\end{array}$} & \multirow[t]{2}{*}{$\begin{array}{c}\text { Total } \\
(n=86)\end{array}$} \\
\hline & Total & Mean & IQR & Total & Mean & IQR & \\
\hline Total affected residents & $\begin{array}{r}3098 \\
(34.2 \%)\end{array}$ & 91.1 & $60-112$ & $\begin{array}{r}5969 \\
(65.8 \%)\end{array}$ & 114.8 & $66-129$ & 9067 \\
\hline ILI symptoms & $\begin{array}{r}452 \\
(34.1 \%)\end{array}$ & 13.3 & $6-17$ & $\begin{array}{r}873 \\
(65.9 \%)\end{array}$ & 16.8 & $9-24$ & 1325 \\
\hline Hospitalisations & $\begin{array}{r}41 \\
(28.3 \%)\end{array}$ & 1.2 & $0-2$ & $\begin{array}{r}104 \\
(71.7 \%)\end{array}$ & 2.0 & $1-3$ & 145 \\
\hline Deaths & $\begin{array}{r}15 \\
(27.8 \%)\end{array}$ & 0.4 & $0-1$ & $\begin{array}{r}39 \\
(72.2 \%)\end{array}$ & 0.8 & $0-1$ & 54 \\
\hline Cases at notification & $\begin{array}{r}293 \\
(36.0 \%)\end{array}$ & 8.6 & $4-11$ & $\begin{array}{r}521 \\
(64.0 \%)\end{array}$ & 10.0 & $6-13$ & 814 \\
\hline Cases post notification & $\begin{array}{r}159 \\
(31.1 \%)\end{array}$ & 4.7 & $0-7$ & $\begin{array}{r}352 \\
(68.9 \%)\end{array}$ & 6.8 & $0.5-10$ & 511 \\
\hline
\end{tabular}

$\mathrm{ILI}=$ influenza-like illness; IQR = interquartile range 
Table 2. Type of influenza reported in NSW residential aged care facilities (RACFs), by neuraminidase prophylaxis use, 1 June 2015 - 31 October 2015

\begin{tabular}{lccc}
\hline Influenza type & $\begin{array}{c}\text { No prophylaxis } \\
(n=34)\end{array}$ & $\begin{array}{c}\text { Prophylaxis } \\
(n=52)\end{array}$ & $\begin{array}{c}\text { Total } \\
(n=86)\end{array}$ \\
\hline RACFs with influenza A only & $16(47.1 \%)$ & $28(53.8 \%)$ & 44 \\
RACFs with influenza B only & $11(32.3 \%)$ & $13(25.0 \%)$ & 24 \\
RACFs with influenza A and B & $7(20.6 \%)$ & $11(21.1 \%)$ & 18 \\
\hline
\end{tabular}

RACFs $=$ residential aged care facilities

Oseltamivir was the NAl used during influenza outbreaks for both prophylaxis and treatment. Prophylactic oseltamivir was used by 52 RACFs (60.5\%) and 58 RACFs (67\%) used oseltamivir for treatment (Figure 1). The average proportion of residents within a facility that received prophylaxis was $51 \%$ (range $0.7-$ 95). Eight RACFs administered NAI prophylaxis only to residents of the initially affected section in the facility.

Eight residents reported discontinuing NAls but reasons for discontinuation were not reported. Of 47 residents who developed an adverse reaction, the type of adverse reaction was recorded for only one (gastrointestinal side effects).

The median time to start NAI prophylaxis after the onset date of the first case was 8.5 days (range 2-23). Most RACFs administered NAls as soon as the medication arrived at their facility; on average, this was 1 day (range 0-3) after NAls had been recommended by the PHU.

The median time for an RACF to first contact the PHU after the onset date of the first case was 6 days in the prophylaxis group (range 0-21) and 7 days in the no prophylaxis group (range 0-22). Ten RACFs contacted the PHU after the onset date of the last case.

The mean vaccination rate for residents in the prophylaxis group was $80.6 \%$ (range 16.3-100), and $74.7 \%$ (range 11.4-98.7) in the no prophylaxis group. Resident vaccination data was not available for six RACFs. The least reported control measure was vaccination of unvaccinated staff $(70 \%$ for the prophylaxis group and $44 \%$ for the no prophylaxis group). Vaccination data were missing for $17 \%$ of all RACF outbreaks.

\section{Discussion}

This observational study examines the use of prophylactic oseltamivir for all reported influenza outbreaks across NSW RACFs during a specified period. Our study demonstrates the challenges of conducting real-world studies in RACFs. These studies do not allow for control of all confounders, and include data collection quality challenges that reflect current practice. These challenges of the RACF setting are noted in other observational studies and randomised controlled trials. ${ }^{12,17}$

Our study has provided valuable information about the use of prophylactic NAls and current practice, within both
RACFs and PHUs. Key areas for quality improvement are described, to support improved use of NAls, where appropriate, in the management of influenza outbreaks in RACFs.

When prescribed, NAI treatment should start within 48 hours of onset of symptoms in adults with laboratoryconfirmed influenza or $\mathrm{ILI}^{2}$, as the efficacy of NAls is highly dependent on the timing of initiation. ${ }^{18}$ In severely ill individuals, the benefits of treatment are reduced when treatment is started after 48 hours of the onset of symptoms. ${ }^{18}$ Similarly, efficacy of NAI prophylaxis is reduced when initiated more than 48 hours after contact with an infectious individual. ${ }^{18,19}$ To optimise the chances of reducing transmission and bringing the outbreak under control, it is recommended that prophylactic NAls be given to all asymptomatic residents and ideally commenced within 24 hours of outbreak recognition. ${ }^{2}$

Delays in outbreak recognition may have contributed to delays in initiation of NAls. Early outbreak recognition is a key step in outbreak control as it provides an opportunity to implement infection control measures, including starting NAIs ${ }^{20,21}$, and has been associated with shorter duration of outbreaks. ${ }^{12}$

Despite our analysis showing that prophylactic NAls are started on average within 1 day of notifying the PHU (and therefore within the recommended 24 hours after outbreak recognition ${ }^{2}$ ), the delay in recognition meant some susceptible residents who may have benefited from prophylactic NAls were likely to have already been exposed to influenza, therefore reducing the effectiveness of prophylaxis. ${ }^{2}$

The reasons for delays in notifying outbreaks to PHUs are not well described in this study. One contributing factor may be that identification of influenza in the elderly is often challenging, particularly in light of existing comorbidities. ${ }^{22}$ Less than $40 \%$ of all cases in this study had reported fever, illustrating the challenges of diagnosing influenza using typical ILI symptoms. Further studies to understand the reasons for notification delays may help provide measures to support RACFs, and improve prompt recognition and notification of influenza outbreaks.

In addition, RACFs may have had multiple general practitioners whose recommendations differed, influencing the time to start NAls. Prescriber behaviour was not explored in this study. It may be that the decision 
to administer prophylaxis was taken because of the severity of the outbreak. Further analysis of these factors, including studies to understand the prescriber decisionmaking process, may provide additional information to support improvements in the use of NAls.

The large proportion of RACFs that administered NAI prophylaxis to fewer than $50 \%$ of residents suggests that strategies are required to improve NAI administration in line with guidelines. The guidelines recommend that, when prescribed, all residents in the RACF are to receive NAI prophylaxis, because of the ease of influenza spreading from one resident to another and from one unit to another. ${ }^{2}$

Despite a NSW Health outbreak line listing template being available, its use was inconsistent across the state. Some RACFs were using outdated templates, either with no field to capture NAI use or specifying antiviral agents no longer in use. The limited reported data on $\mathrm{NAI}$ compliance and adverse reactions experienced also highlight data reporting challenges. Ensuring that current and consistent data fields are used in line listings can support the appropriate use of NAIs, improve data collection processes and support future evaluation.

NAls are not recommended independent of other outbreak control measures, such as high vaccination rates for residents and staff. It is well known that staff influenza vaccination rates are often low in these settings $^{23}$, and this is reinforced by the lack of staff vaccination data reported in this study. RACF vaccination policies and other strategies to support uptake of influenza vaccine by staff are therefore critical, since unvaccinated staff are more likely to introduce influenza to a facility, and to spread the infection once it is introduced. ${ }^{23,24}$

Feedback from PHU staff highlighted the value of early engagement with RACFs before the influenza period, reporting that this supported the reporting process and provided an opportunity for RACF staff education. Continuing early collaboration between RACFs and PHUs may support future influenza outbreak control.

\section{Limitations}

Our results are limited by the challenges of conducting observational studies in the RACF setting, including not being able to control for all confounders, and data collection quality challenges.

Because the effectiveness of NAls is dependent on how they are administered at an individual level, it is important to have information about duration of therapy or reason for cessation. However, incomplete line listings and NAI compliance data meant it was not possible to conclusively assess how NAls were used for each individual resident. Further, more comprehensive information in the line listings could have highlighted any discrepancies in influenza case definitions between facilities.
Availability of staff data, including ratio of staff to residents, number of staff with influenza or ILI, and number of staff who commenced oseltamivir for treatment or prophylaxis, may have provided additional information about outbreaks and staff impact on implementing NAls. Access to facility maps may have helped us determine the extent of influenza transmission between areas and the impact of different sizes and types of facilities.

\section{Conclusion}

Influenza outbreaks in RACFs are common and place a burden on residents, staff and the health system. The use of NAI prophylaxis differs across NSW RACFs and strategies that support improvements in their use are recommended. This includes earlier initiation of NAls, improved resident coverage where appropriate, improved reporting of outbreaks by RACFs and the use of appropriate line listings. In addition, early outbreak recognition, increased staff vaccination and continued collaboration with PHUs may support the management and control of influenza outbreaks in RACFs.

\section{Peer review and provenance}

Externally peer reviewed, not commissioned.

\section{Competing interests}

None declared.

\section{Author contributions}

ST, RG and WM designed the study. WM reviewed and cleaned the data. WM and $\mathrm{DH}$ analysed the data, and $\mathrm{DH}, \mathrm{WM}, \mathrm{ST}, \mathrm{RG}$ and VS interpreted the data. WM drafted the manuscript and ST, DH, RG, VS and WM were responsible for editing. All of the authors approved the manuscript for publication.

\section{References}

1. Heymann DL, editor. Control of communicable diseases manual. 20th ed. Washington DC: American Public Health Association; 2014.

2. Communicable Diseases Network of Australia. Guidelines for the prevention, control and public health management of influenza outbreaks in residential care facilities in Australia. Canberra: Commonwealth of Australia; 2017 [cited 2017 Mar 30]. Available from: www.health.gov.au/ internet/main/publishing.nsf/Content/cdna-flu-guidelines.htm

3. Australian Technical Advisory Group on Immunisation (ATAGI). The Australian immunisation handbook. 10th ed (2017 update). Canberra: Australian Government Department of Health; 2017 [cited 2019 Jan 16]. Available from: immunisationhandbook.health.gov.au 
4. Jefferson T, Di Pietrantonj C, Al-Ansary LA, Ferroni E, Thorning S, Thomas RE. Vaccines for preventing influenza in the elderly. Cochrane Database Syst Rev. 2010;17(2):CD004876.

5. NSW Government. Public Health Act 2010. Sydney: NSW Government; 2018 [cited 2019 Jan 16]. Available from: www.legislation.nsw.gov.au/\#/view/act/2010/127

6. Fiore AE, Fry A, Shay D, Gubareva L, Bresee J, Uyeki T, Centers for Disease Control and Prevention (CDC). Antiviral agents for the treatment and chemoprophylaxis of influenza: recommendations of the Advisory Committee on Immunization Practices (ACIP). MMWR Recomm Rep. 2011;60(1):1-24.

7. World Health Organization. WHO guidelines for pharmacological management of pandemic influenza A(H1N1) 2009 and other influenza viruses. Geneva: WHO; 2010 [cited 2017 Mar 30]. Available from: www.who.int/csr/resources/publications/swineflu/h1n1_ guidelines_pharmaceutical_mngt.pdf

8. Jefferson T, Jones MA, Doshi P, Del Mar CB, Hama R, Thompson MJ, et al. Neuraminidase inhibitors for preventing and treating influenza in healthy adults and children. Cochrane Database Syst Rev. 2014;10(4):CD008965.

9. Okoli GN, Otete HE, Beck CR, Nguyen-Van-Tam JS. Use of neuraminidase inhibitors for rapid containment of influenza: a systematic review and meta-analysis of individual and household transmission studies. PloS One. 2014;9(12):e113633

10. Bowles SK, Lee W, Simor AE, Vearncombe M, Loeb M, Tamblyn S, et al. Use of oseltamivir during influenza outbreaks in Ontario nursing homes, 1999-2000. J Am Geriatr Soc. 2002;50(4):608-16.

11. Parker R, Loewen N, Skowronski D. Experience with oseltamivir in the control of a nursing home influenza $\mathrm{B}$ outbreak. Can Commun Dis Rep. 2001;27(5):37-40.

12. Ye M, Jacobs A, Khan MN, Jaipaul J, Oda J, Johnson M, et al. Evaluation of the use of oseltamivir prophylaxis in the control of influenza outbreaks in long-term care facilities in Alberta, Canada: a retrospective provincial database analysis. BMJ Open. 2016;6(7):e011686.

13. Harper SA, Bradley JS, Englund JA, File TM, Gravenstein S, Hayden FG, et al. Seasonal influenza in adults and children--diagnosis, treatment, chemoprophylaxis, and institutional outbreak management: clinical practice guidelines of the Infectious Diseases Society of America. Clin Infect Dis. 2009;48(8):1003-32.
14. Aoki FY, Allen UD, Stiver HG, Evans GA. The use of antiviral drugs for influenza: guidance for practitioners 2012/2013. Can J Infect Dis Med Microbiol. 2012;23(4):e79-92.

15. European Centre for Disease Prevention and Control. Seasonal influenza vaccination and antiviral use in Europe: overview of vaccination recommendations and coverage rates in the EU Member States for the 2013-14 and 2014-15 influenza seasons. Stockholm: ECDC; 2016 [cited 2016 Sep 16]. Available from: www.ecdc.europa.eu/en/publications/Publications/ Seasonal-influenza-vaccination-antiviral-use-europe.pdf

16. Merritt T, Hope K, Butler M, Durrheim D, Gupta L, Najjar Z, et al. Effect of antiviral prophylaxis on influenza outbreaks in aged care facilities in three local health districts in New South Wales, Australia, 2014. Western Pac Surveill Response J. 2016;7(1):14-20.

17. Booy R, Lindley RI, Dwyer DE, Yin JK, Heron LG, Moffatt $\mathrm{CR}$, et al. Treating and preventing influenza in aged care facilities: a cluster randomised controlled trial. PLoS One. 2012;7(10):17.

18. Aoki FY, Macleod MD, Paggiaro P, Carewicz O, El Sawy A, Wat C, et al. Early administration of oral oseltamivir increases the benefits of influenza treatment. J Antimicrob Chemother. 2003;5(1):123-9.

19. The Academy of Medical Sciences. Use of neuraminidase inhibitors in influenza. London: The Academy of Medical Sciences and Wellcome Trust; 2015 [cited 2016 Sep 16]. Available from: www.acmedsci. ac.uk/file-download/38069-561595082cd83.pdf

20. Seale H, Weston KM, Dwyer DE, Mengzhi Z, Allchin L, Booy $\mathrm{R}$, et al. The use of oseltamivir during an influenza B outbreak in a chronic care hospital. Influenza Other Respir Viruses. 2009;3(1):15-20.

21. Monto AS, Rotthoff J, Teich E, Herlocher ML, Truscon R, Yen $\mathrm{HL}$, et al. Detection and control of influenza outbreaks in well-vaccinated nursing home populations. Clin Infect Dis. 2004;15;39(4):459-64.

22. Talbot $\mathrm{H}$, Falsey $A$. The diagnosis of viral respiratory disease in older adults. Clin Infect Dis. 2010;50:747-51.

23. Guy R, Di Natale R, Kelly HA, Lambert SB, Tobin S, Robinson PM, et al. Influenza outbreaks in aged-care facilities: staff vaccination and the emerging use of antiviral therapy. Med J Aust. 2004;180(12):640-42.

24. Bush KA, McAnulty J, McPhie K, Reynolds R, Boomer M, Clarkson L, et al. Antiviral prophylaxis in the management of an influenza outbreak in an aged care facility. Commun Dis Intell Q Rep. 2004;28(3):396-400.

\section{Copyright: (c)}

(C) 2019 Meshreky et al. This article is licensed under the Creative Commons Attribution-NonCommercial-ShareAlike 4.0 International Licence, which allows others to redistribute, adapt and share this work non-commercially provided they attribute the work and any adapted version of it is distributed under the same Creative Commons licence terms. See: www.creativecommons.org/licenses/by-nc-sa/4.0/ 